\title{
Safe Distributed Control of Wireless Power Transfer Networks
}

\author{
Kasım Sinan Yıldırım, Ruggero Carli, Member, IEEE, and Luca Schenato, Fellow, IEEE
}

\begin{abstract}
Wireless power transfer networks (WPTNs) are composed of dedicated energy transmitters that charge energy receivers via radio frequency waves. A safe-charging WPTN should keep electromagnetic radiation below pre-determined limits meanwhile maximizing the transmitted power. In this article we consider this requirement as an optimization problem: the maximization of harvested power by ERs subject to the electro-magnetic safety constraints. In order to provide an approximated solution to this problem, we introduce a dual ascent-like distributed charging algorithm that enables energy transmitters to work without global information and satisfy safety constraints asymptotically. We provide an in-depth theoretical analysis of our algorithm which is supported by numerical simulations.
\end{abstract}

Index Terms-Wireless Power Transfer, Electromagnetic Radiation, Distributed Optimization

\section{INTRODUCTION}

Using batteries to power millions of interconnected devices forming today's Internet of Things (IoT) is not feasible since batteries increase the weight, the cost, the maintenance overhead and the ecological footprint of the embedded hardware [1]. Fortunately, the standalone and sustainable operation of the embedded devices without any batteries is becoming more feasible thanks to advancements in ambient energy harvesting. In particular, by means of radio frequency (RF) energy harvesting, a new class of embedded devices that can sense, compute and communicate using the harvested RF energy only has already emerged - so called the RFpowered computers [2]. These computers have very interesting future applications, e.g. battery-less sensing using wireless-ly powered cameras has already been demonstrated in [3].

In order to enable sustainable operation and charge RFpowered computers, a dedicated network of electromagnetic wave energy emitters should be deployed. Such networks, namely wireless power transfer networks (WPTNs) [4], are composed of several energy transmitter nodes (ETs) that control their power levels in order to charge nearby energy receiver nodes (ER) collaboratively to maximize the total transmitted power, and in turn to charge them as quickly as possible. However, WTPNs should also ensure that humans are not exposed to excessive electromagnetic radiation (EMR) [1]. Therefore, a safe-charging WPTN should comply with the $\mathrm{RF}$ exposure regulations [5].

Kasım Sinan Yıldırım is with the Department of Computer Engineering, Ege University, İzmir, Turkey and the Embedded Software group, TU Delft, The Netherlands. Ruggero Carli and Luca Schenato are with the Department of Information Engineering, University of Padova, Italy.

\section{A. Problem Statement}

In this article, we assume that there are sensor nodes deployed at specific locations so that ETs can communicate with these sensors and obtain their measurements in order to detect overexposure to EMR. Our focus is to answer the question of how to maximize the total transmitted power to ERs meanwhile complying with the RF exposure regulations? This defines an optimization problem with safety constraints that dictates the measured values at sensor locations not to exceed a pre-defined EMR threshold value. Unfortunately, it is not feasible to obtain a one-shot centralized solution to this optimization problem due to the following reasons. First, a centralized solution uses a mathematical model for the sensor EMR measurements rather than incorporating the actual measured values into the calculations. However, the real measurements cannot be represented perfectly using a theoretical radio wave propagation model since the transmitted power is inherently random due to environmental effects [1]. Second, ERs and sensors as well as ETs might not be stationary. In order to keep the charging network safe despite changed positions or addition/removal of the new devices, the whole network state should be collected at a centralized entity to recalculate the optimal power levels satisfying safety constraints. Considering these facts, an online distributed solution that is reactive against network dynamics and does not require global network information is required in order to meet safety constraints effectively in practice.

\section{B. Contributions}

In this article, we introduce a feedback-based dual ascentlike distributed charging algorithm to provide an approximated solution to the aforementioned optimization problem: ETs maximize the total transmitted power meanwhile satisfying safety constraints without global information. In particular:

- Our algorithm allows ETs to communicate only with the sensor nodes within their communication range-ETs communicate neither with other ETs nor ERs to update their power levels, leading to a simplistic implementation with low message complexity.

- Each ET uses actual measurements from sensors-since the measurements of the sensor nodes are not estimated by using theoretical RF propagation models, the proposed algorithm satisfies the safety constraints asymptotically.

- We provide an in-depth theoretical analysis of the proposed algorithm supported by numerical simulations.

The rest of this article is organized as follows. Section II presents the state of the art and Section III describes the system 
model and problem statement. We provide an approximated optimal solution to the aforementioned optimization problem in Section IV. In Section V, we describe how the approximated algorithm can be implemented in a distributed manner. We provide numerical simulations in Section VI. Finally, we conclude and present future work in Section VII.

\section{RELATED WORK}

The prior studies our study builds upon can be divided into the following categories:

a) Wireless Charging: The methods for wireless power transfer can be classified into the non-radiative techniques that can charge devices at short distances and radiative techniques that typically use RF-waves as an energy delivery medium to charge devices at long distances [6]. Even though these techniques are not new, several researchers started to draw more attention to RF-based charging due to the emergence of ultra low-power embedded systems [7]. The power requirements of such devices are only a few $\mu \mathrm{Ws}$ that enabled battery-less operation using only the harvested electromagnetic energy [8]. RF-based charging can be used to charge many receivers simultaneously by means of its broadcast nature and it is suitable for mobile applications [1]. Several efforts have still been devoted towards to increase the efficiency of RF energy harvesting, e.g. [9].

b) RF-Based Charging Networks: The provision of energy to the embedded devices via dedicated network of RFenergy transmitters has been demonstrated before[4]. There are several recent research efforts in this domain focused on the scalability of these networks in terms of total transmitted power [10], minimization of the charging delay [11], charging control of the energy transmitters to maximize the lifetime of the network and minimize energy outage [12], [13],

c) RF-Based Charging Safety: The radiation safety during RF-based charging is generally overlooked in the current state of the art. It is generally accepted that being exposed to radiation is considered as a threat for human health [14] and the exposure regulations define the limits for the radiated power from electronic devices [15]. As emphasized in [16], satisfying radiation constraints on every point on a $2 \mathrm{D}$ area is an NP-hard problem. To this end, [17] and [16] aimed at providing efficient centralized solutions for the radiationconstrained wireless power transfer optimization on a predefined deployment area. To the best of our knowledge, [18] provided the only distributed solution for the aforementioned optimization problem. The algorithm proposed in this study is composed of several phases: (i) a distributed redundant constraint reduction algorithm is executed; (ii) the deployment area is splitted into several small squares so that ETs can employ linear programming (LP) locally; (iii) all the local LP solutions are merged to obtain the global optimal solution. These phases requires several computation steps and also communication rounds among the ETs.

d) Distributed Optimization: In most of the existing approaches, e.g. distributed subgradient methods [19], Lagrangian-based methods [20], consensus-based methods [21], and distributed linear programming [22], each agent

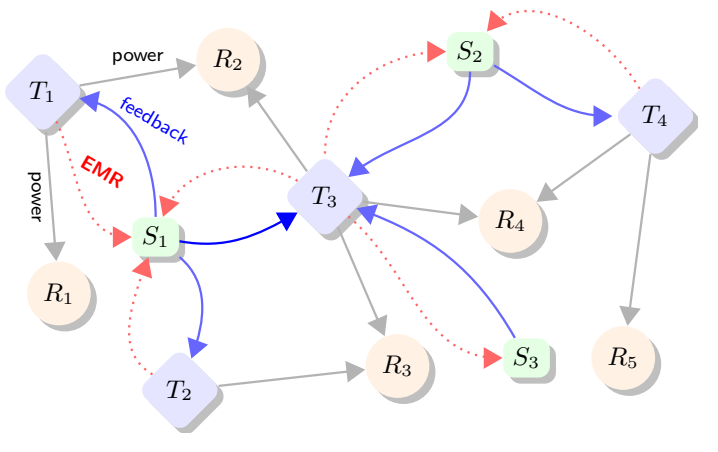

Fig. 1. A representative graph-theoretical view of a WPTN. Energy transmitters are denoted by $T_{i}$, energy receiver devices are denoted by $R_{j}$ and sensor nodes are denoted by $S_{k}$.

computes the whole global minimizer-reasonable when the number of decision variables is independent of the number of agents involved. However, in our context the number of decision variables scale with the number of agents, thus giving rise to an unscalable solution. In recent alternative algorithms, referred as partition-based, each agent is required to compute only a subset of the decision variables [23], [24], [25]. Nonetheless, they are not directly applicable to our specific problem since they are either developed for unconstrained problems [23], or admit only equality constraints [24], [25].

e) Our Difference From Prior Studies: In this article, we propose a distributed algorithm which is fundamentally different from the existing safe-charging algorithms. First, all of the aforementioned studies use a representative RF propagation model to estimate the EMR value at particular locations. However, estimations might not represent the actual measurements, that might lead safety violations in practice. On the contrary, we consider a different system model that is composed of sensor nodes at particular locations sending actual EMR measurement feedbacks. As a consequence, the algorithm proposed in this article guarantees safety constraints asymptotically. Second, our algorithm does not require communication among ETs. Rather, it relies on the feedbacks from the sensors in order to adjust the power levels and satisfy safety constraints. Computations pertaining to this adjustment are simple and do not include any complex operations.

Finally, we would like to mention that our work builds upon the prior work in [26], [27]. In [27], we presented a different algorithm which is based on the method of multipliers but we did not provide theoretical analysis and limited ourselves to only numerical results. In [26] we proposed an algorithm which, as the one described in this article, is based on dualascent approach. However, in [26], in order to force the physical constraints related the amount of power that can be transmitted, we modified the functional cost adding suitable barrier functions-which led to some disadvantages which are discussed in details in Section IV Remark IV.2. This article is inspired by the ideas from these works, presents a different algorithm together with in-depth theoretical analysis supported by numerical simulations. 


\section{Modeling AND PROBlem Formulation}

We represent a WPTN as a graph which is composed of different types of nodes representing RF-based energy transmitters (ETs), energy receivers (ERs) equipped with RF energy harvesters, and sensor nodes (SNs) as depicted in Fig. 1. We indicate these nodes with the sets $\mathcal{T}=\left\{T_{1}, T_{2}, \ldots, T_{N}\right\}$, $\mathcal{R}=\left\{R_{1}, R_{2}, \ldots, R_{K}\right\}$, and $\mathcal{S}=\left\{S_{1}, S_{2}, \ldots, S_{M}\right\}$, respectively. Their location in space is defined by the variable $x \in \mathbb{R}^{3}$ instantiated as $x_{T_{i}}$ to indicate the location of $i$-th transmitter node (similarly for $x_{R_{i}}$ and $x_{S_{i}}$ ). Each ET can charge ERs within its wireless power transmission range. The output power of $T_{i}$ is denoted by $P_{i}$ which is limited by a maximum power level denoted by $P_{\max }$ so that

$$
0 \leq P_{i} \leq P_{\max }, \quad i \in \mathcal{T},
$$

where with a little abuse of notation $P_{i}=P_{T_{i}}$. We assume for simplicity that each ET can adjust its power $P_{i}$ at any value between these two extremes, i.e. we neglect possible discrete power levels. We also assume that the power $P$ generated at a location $x$ arrives at different location $y$ attenuated by a power attenuation factor $0 \leq \kappa(x, y) \leq 1$, i.e., the power at location $y$ is given by $P_{x, y}=P \kappa(x, y)$. For sake of simplicity we assume an isotropic attenuation within a certain radius and null outside:

$$
\kappa(x, y)= \begin{cases}\frac{\gamma}{\|x-y\|^{2}+\gamma} & \text { if }\|x-y\| \leq r \\ 0 & \text { otherwise }\end{cases}
$$

where $\gamma$ and $r$ are suitable positive constants. In practice, such coefficient depends specifically on the environment (multiple reflections, occlusions, fading, ...) and therefore the previous expression should be considered only as a crude estimation of the true power attenuation factor. The power attenuation factor is then used to model the power received by the ERs and power measured by the SNs. More specifically we define the following power transfer matrix $A \in \mathbb{R}^{K \times N}$ and sensed power matrix $B \in \mathbb{R}^{M \times N}$ whose $i-j$ entries are given by:

$$
\begin{aligned}
& A_{i j}:=\alpha \kappa\left(x_{R_{i}}, x_{T_{j}}\right), \quad i=1, \ldots, K \quad j=1, \ldots, N \\
& B_{i j}:=\beta \kappa\left(x_{S_{i}}, x_{T_{j}}\right), \quad i=1, \ldots, M \quad j=1, \ldots, N
\end{aligned}
$$

where $\alpha$ and $\beta$ are suitable positive constant which model the power conversion factor and the power sensor scaling, respectively. Based on these matrices we define the following neighbours sets

$$
\begin{array}{ll}
\mathcal{T}_{i}^{\mathcal{R}}=\left\{j \in \mathcal{T} \mid A_{i j}>0\right\}, & \mathcal{R}_{i}^{\mathcal{T}}=\left\{j \in \mathcal{R} \mid A_{j i}>0\right\} \\
\mathcal{T}_{i}^{\mathcal{S}}=\left\{j \in \mathcal{T} \mid B_{i j}>0\right\}, & \mathcal{S}_{i}^{\mathcal{T}}=\left\{j \in \mathcal{S} \mid B_{j i}>0\right\}
\end{array}
$$

where $\mathcal{T}_{i}^{\mathcal{R}}$ corresponds to the set of ETs which contribute to the power received by the $i$-th ER, $\mathcal{R}_{i}^{\mathcal{T}}$ corresponds to the set of ERs which receives power from the $i$-th ET, $\mathcal{T}_{i}^{\mathcal{S}}$ corresponds to the set of ETs which contribute to the power measured by the $i$-th $\mathrm{SN}$, and $\mathcal{S}_{i}^{\mathcal{T}}$ corresponds to the set of SNs which measure power from the $i$-th ET.

We will assume that the communication graph is consistent with these sets, i.e. the $i$-th ET can charge the $j$-th ER if and only if $A_{j i}>0$, and similarly the $i$-th ET and $\ell$-th SN can communicate with each other if and only if $B_{\ell i}>0$. This assumption is not unrealistic since the communication graph and the power transmission graph in WPTN basically coincide.

Under the assumption that the power transmitted from multiple sources and received at a certain location is additive, we can define the power received by the $i$-th ER, $\eta_{i}$, and the power measured by the $\ell$-th $\mathrm{SN}, \delta_{\ell}$ is given by

$$
\begin{aligned}
\eta_{i} & :=\sum_{j=1}^{N} A_{i j} P_{j}=\sum_{j \in \mathcal{T}_{i}^{\mathcal{R}}} A_{i j} P_{j}, \quad i=1, \ldots, K \\
\delta_{\ell} & :=\sum_{j=1}^{N} B_{\ell j} P_{j}=\sum_{j \in \mathcal{T}_{i}^{\mathcal{S}}} B_{\ell j} P_{j}, \quad i=1, \ldots, M
\end{aligned}
$$

\section{A. Problem Formulation}

The objective of the WPTN is to charge ERs as fast as possible meanwhile ensuring that the EMR values measured by each sensor deployed at particular points are always smaller than a pre-defined EMR threshold, $\delta_{\max }$, i.e.

$$
\delta_{\ell} \leq \delta_{\max }, \quad \ell=1, \ldots, M
$$

As a possible metrics for optimality, we pick the sum of the received power by all the ERs, i.e. the total received power, $P_{\text {Total }}$ :

$$
P_{\text {Total }}=\sum_{i=1}^{N} \eta_{i}=\sum_{i=1}^{N} \sum_{j \in \mathcal{T}_{i}^{\mathcal{R}}} A_{i j} P_{j}
$$

Now let us define the following vectors $P=\left[P_{1} \cdots P_{N}\right]^{T}, \eta=$ $\left[\eta_{1} \cdots \eta_{K}\right]^{T}, \delta=\left[\delta_{1} \cdots \delta_{M}\right]^{T}, \mathbf{1}=[1 \cdots 1]^{T} \in \mathbb{R}^{N}, \mathbf{0}=$ $[0 \cdots 0]^{T} \in \mathbb{R}^{N}$. Noticing that $\eta=A P, \delta=B P$, and $J=\mathbf{1}^{T} \eta=\mathbf{1}^{T} A P$, we can write the optimization problem as

$$
\begin{array}{cc}
\max _{P} & \mathbf{1}^{T} A P \\
\text { s.t. } & B P \leq \delta_{\max } \mathbf{1} \\
& \mathbf{0} \leq P \leq P_{\max } \mathbf{1}
\end{array}
$$

where the inequalities are to be interpreted component-wise. More specifically, (5) corresponds to the total received power, the inequality (6) can be interpreted as a soft constraint, since violation of power limit at the sensors can be allowed occasionally, while inequality (7) is hard constraint since these bounds cannot be physically violated. The optimization problem just defined is clearly a Linear Program (LP) optimization problem which could be easily solved via a centralized solver as long as the matrices $A, B$ are known. This is however problematic in a WPTN framework. To overcome this problem in the next section we will propose a feedback-based distributed optimization solution which has several advantages: it does not require any global coordination among the nodes, it exploits the sparsity of matrices $A$ and $B$ by performing only local communication, it uses the measurements of the sensor to asymptotically guaranteeing inequality (6) even if the matrices $A$ and $B$ are not perfectly known.

Remark III.1 The matrices $A$ and $B$ can be time-varying if the nodes are moving, i.e. $x_{R_{i}}, x_{S_{i}}$ and $x_{T_{j}}$ are changing. In the following sections, we will consider the static scenario where matrices $A$ and $B$ do not change over time. In practice, 
we expect that the convergence rate of the algorithm proposed in Section $V$ to be much faster than the rate of the change in the network topology. This scenario is explored in the Numerical Simulations Section VI.

\section{AN APPROXIMATED OPTIMAL SOLUTION}

We will start by showing how a standard primal-dual ascent algorithm cannot be applied in our scenario, we will later show how an approximate optimization problem can address this limitation, and finally we provide some parameter optimization design procedures and characterisation of optimality.

\section{A. Limitation of naive primal-dual iterative algorithm}

Observe that the LP problem (5)-(7) is equivalent to the following problem:

$$
\begin{array}{rc}
P^{*}:=\arg \min _{P \in \mathcal{P}} \quad J(P):=-a^{T} P \\
\text { s.t. } \quad B P \leq \delta_{\max } \mathbf{1}
\end{array}
$$

where $\mathcal{P}:=\left\{P \mid \mathbf{0} \leq P \leq P_{\max } \mathbf{1}\right\}$ is a closed convex set, more specifically a hypercube, and $a=\left[a_{1} \cdots a_{N}\right]^{T}$ and $a_{i}=\sum_{j=1}^{K} A_{j i}=\sum_{j \in \mathcal{R}_{i}^{\mathcal{T}}} A_{j i}$. The optimization problem above is clearly feasible since $P=\mathbf{0} \in \mathcal{P}$ satisfies the EMR constraints. However, it might not have a unique minimizer $P^{*}$ being the cost function $-J(P)=-a^{T} P$ simply convex. A standard approach in constrained convex optimization is to consider the corresponding Lagrangian

$$
\mathcal{L}(P, \lambda)=-a^{T} P+\lambda^{T}\left(B P-\delta_{\max } \mathbf{1}\right)
$$

where $\lambda \in \mathbb{R}^{M}$ and dual function is given by

$$
q(\lambda)=\min _{P \in \mathcal{P}} \mathcal{L}(P, \lambda)
$$

and its maximizer is written as

$$
\lambda^{*}:=\arg \max _{\lambda \geq 0} q(\lambda)
$$

From convex optimization theory it also follows that there is no duality gap between the primal and the dual problem, i.e.:

$$
q\left(\lambda^{*}\right)=-a^{T} P^{*}
$$

At this point, one might be tempted to apply a standard primal-dual coupled iterative algorithm to find a solution to both the primal and dual problem as follows:

$$
\begin{aligned}
P^{k+1} & =\arg \min _{P \in \mathcal{P}} \mathcal{L}\left(P, \lambda^{k}\right) \\
\lambda^{k+1} & =\max \left\{\mathbf{0}, \lambda_{k}+\rho_{k}\left(B P^{k+1}-\delta_{\max } \mathbf{1}\right)\right\}
\end{aligned}
$$

where $\rho_{k}$ is a (possibly time-varying) step size for the dual ascent, and the max operator has to be interpreted componentwise. The previous algorithm however does not guarantee to provide an optimal solution of the primal problem, the problem being that $P_{k+1}$ might not be unique since $\mathcal{L}\left(P, \lambda^{k}\right)$ is simply convex in $P$. In fact, although it can be shown that for a suitable decreasing step-size $\rho_{k}$, we have $\lambda^{k} \rightarrow \lambda^{*}$, this does not help to guarantee $P_{k} \rightarrow P^{*}$ if $\arg \min _{P \in \mathcal{P}} \mathcal{L}\left(P, \lambda^{*}\right)$ does not provide a unique solution. To show this let us consider the scenario where only one ET, one ER, and one SN are present. Also assume that $A=B=P_{\max }=1$ and $0<\delta_{\max }<1$. Clearly, in this scenario $P^{*}=\delta_{\max }$ and it is unique. Moreover, by explicit computation of $q(\lambda)$ it is possible to derive that $\lambda^{*}=1$ which gives $\mathcal{L}\left(P, \lambda^{*}\right)=-\delta_{\text {max }}$, therefore $P^{k+1}=\arg \min _{P \in \mathcal{P}} \mathcal{L}\left(P, \lambda^{*}\right)$ does not provide the minimizer of primal problem-which is our interest.

Remark IV.1 An alternative solution would be the use of augmented Lagrangian strategies such as ADMM [20]. However, these are suited for optimization problems with equality constraints. One possibility to circumvent this problem is to add additional variables $z_{i}$, one for each sensor node, and solve the following optimization problem:

$$
\begin{array}{rc}
\left(P^{*}, z^{*}\right):=\arg \min _{P \in \mathcal{P}, z \geq 0} \quad J(P):=-a^{T} P \\
\text { s.t. } & \delta_{\max } \mathbf{1}-B P=z
\end{array}
$$

where $z=\left[z_{1} \cdots z_{M}\right]^{T}$ and then apply ADMM. Nonetheless, the complexity of the algorithm in terms of computation and communication will increase and its understanding requires a dedicated analysis which is beyond the scope of this work.

As such, we propose to approximate the objective function by adding a regularization term which would make the primal problem strongly convex as shown in the next section.

\section{B. Approximated optimization problem}

Consider the following approximated optimization problem:

$$
\begin{array}{rc}
P_{\theta}^{*}:=\arg \min _{P \in \mathcal{P}} & J_{\theta}(P):=-a^{T} P+\frac{\theta}{2}\left\|P-\frac{P_{\max }}{2} \mathbf{1}\right\|^{2} \\
\text { s.t. } & B P \leq \delta_{\max } \mathbf{1}
\end{array}
$$

and the corresponding Lagrangian and dual functions:

$$
\begin{gathered}
\mathcal{L}_{\theta}(P, \lambda)=-a^{T} P+\lambda^{T}\left(B P-\delta_{\max } \mathbf{1}\right)+\frac{\theta}{2}\left\|P-\frac{P_{\text {max }}}{2} \mathbf{1}\right\|^{2} \\
q_{\theta}(\lambda)=\min _{P \in \mathcal{P}} \mathcal{L}_{\theta}(P, \lambda)
\end{gathered}
$$

The addition of the regularization term $\frac{\theta}{2}\left\|P-\frac{P_{\max }}{2} \mathbf{1}\right\|^{2}$ basically bias each transmitter towards the mid power level $\frac{P_{\max }}{2}$. Clearly, the approximated optimization problem will provide a suboptimal solution, however we expect by continuity arguments that

$$
\lim _{\theta \rightarrow 0} J_{\theta}\left(P_{\theta}^{*}\right)=J\left(P^{*}\right) .
$$

This fact is confirmed by the bound on the difference between $J_{\theta}\left(P_{\theta}^{*}\right)$ and $J\left(P^{*}\right)$, which is provided in the following proposition.

Proposition IV.1 Let $P^{*}$ and $P_{\theta}^{*}$ be defined as in (8) and (9), respectively. Then,

$$
0 \leq J_{\theta}\left(P_{\theta}^{*}\right)-J\left(P^{*}\right) \leq \frac{\theta N}{8} P_{\max }^{2}
$$

Proof: Since $J_{\theta}(P) \geq J(P)$ for all $P$, it easily follows that $J_{\theta}\left(P_{\theta}^{*}\right) \geq J\left(P^{*}\right)$.

Now, observe that for any $P \in \mathcal{P}$ it holds that

$$
\left\|P-\left(P_{\max } / 2\right) \mathbf{1}\right\|^{2} \leq(N / 4) P_{\max }^{2} .
$$


Since for any $P \in \mathcal{P}$ such that $B P \leq \delta_{\max } 1$, it holds also that $J_{\theta}\left(P_{\theta}^{*}\right) \leq J_{\theta}(P)$, we can write that

$$
\begin{aligned}
J_{\theta}\left(P_{\theta}^{*}\right) & \leq J_{\theta}\left(P^{*}\right) \\
& \leq-a^{T} P^{*}+\frac{\theta}{2}\left\|P^{*}-\left(P_{\max } / 2\right) \mathbf{1}\right\|^{2} \\
& \leq-a^{T} P^{*}+\frac{\theta}{2} \frac{N}{4} P_{\max }^{2} \\
& \leq J\left(P^{*}\right)+\frac{\theta N}{8} P_{\text {max }}^{2} .
\end{aligned}
$$

This concludes the proof.

Based on the previous result, one would be tempted to choose a very small value for $\theta$, but as it will be shown later, this gives rise to a slower rate of convergence of $P^{k} \rightarrow P_{\theta}^{*}$.

Remark IV.2 A number of remarks are now in order:

(i) Although different bias power levels could be chosen, as for example $\frac{\theta}{2}\|P\|^{2}$ or $\frac{\theta}{2}\left\|P-P_{\max } \mathbf{1}\right\|^{2}$, the choice provided above, is the one that, in general, reduces the approximation gap $J\left(P_{\theta}^{*}\right)-J\left(P^{*}\right)$, as it will be shown later.

(ii) Alternative regularization terms can be used. For example, a typical choice is to use log-barriers by including the regularization term

$$
-\theta\left(\sum_{i=1}^{N} \log P_{i}+\sum_{i=1}^{N} \log \left(P_{\max }-P_{i}\right)\right)
$$

as done in [26], which has also the benefit to automatically enforce the constraint $P \in \mathcal{P}$ and therefore can be removed from the optimization problem. However, this regularization term has three disadvantages: the first being that, in general, the approximation gap $J\left(P_{\theta}^{*}\right)-J\left(P^{*}\right)$ is larger, the second is that the transmitter are never allowed to fully exploit maximum power or be idles since $\mathbf{0}<P_{\theta}^{*}<P_{\max } \mathbf{1}$, and the computation of the optimal power level at each iteration $P^{k+1}$ is more involved.

(iii) Since the Lagrangian $\mathcal{L}_{\theta}(P, \lambda)$ is quadratic in $P$, its minimizer is unique and can be computed in closed form.

(iv) The parameter $\theta$ is proportional to the error of the approximation and therefore we would like to select it sufficiently small. If we assume that during normal operation, each receiver receives, on average, an amount of power proportional to the total maximum power transmitted by all sensors $N P_{\max }$, i.e. $J(P *) \approx \ell N P_{\max }$, where $\ell$ is in the order of unity, then the bound on the maximum relative error induced by $\theta$ is given by

$$
\frac{\left|J\left(P^{*}\right)-J_{\theta}\left(P_{\theta}^{*}\right)\right|}{\left|J\left(P^{*}\right)\right|} \stackrel{\sim}{\simeq} \frac{\theta}{8 \ell} P_{\max } .
$$

Therefore to have at worst a $1 \%$ error, we should pick $\theta \approx 0.01 \frac{8 \ell}{P_{\max }}$.

Next, we prove important properties for the approximated optimization problem.

Proposition IV.2 Let $P^{\theta}(\lambda):=\arg \min _{P \in \mathcal{P}} \mathcal{L}_{\theta}(P, \lambda)$ where $\lambda \in \mathbb{R}^{M}, \theta>0$. Then the vector $P^{\theta}(\lambda)=\left[P_{1}^{\theta}(\lambda) \cdots P_{N}^{\theta}\right]^{T}$ is unique and it is given by:

$$
P^{\theta}(\lambda)=\operatorname{proj}_{\mathcal{P}}\left(\frac{P_{\text {max }}}{2} \mathbf{1}+\frac{1}{\theta}\left(a-B^{T} \lambda\right)\right)
$$

where $\operatorname{proj}_{\mathcal{P}}$ is the projection operator on the convex set $\mathcal{P}$, or equivalently:

$$
P_{i}^{\theta}(\lambda)=h\left(c_{i}\right)= \begin{cases}0 & \text { if } c_{i}>\theta \frac{P_{\max }}{2} \\ \frac{P_{\max }}{2}-\frac{c_{i}}{\theta} & \text { if }-\theta \frac{P_{\max }}{2} \leq c_{i} \leq \theta \frac{P_{\max }}{2} \\ P_{\max } & \text { if } c_{i}<-\theta \frac{P_{\max }}{2}\end{cases}
$$

where $c_{i}=\sum_{j \in \mathcal{S}_{i}^{\mathcal{T}}} B_{j i} \lambda_{j}-a_{i}=\sum_{j \in \mathcal{S}_{i}^{\mathcal{T}}} B_{j i} \lambda_{j}-\sum_{j \in \mathcal{R}_{i}^{\mathcal{T}}} A_{j i}$.

Proof: The Lagrangian can be written as

$$
\mathcal{L}_{\theta}(P, \lambda)=\sum_{i=1}^{N}\left(\left(-a_{i}+\sum_{j \in \mathcal{S}_{i}} B_{j i} \lambda_{j}\right) P_{i}+\frac{\theta}{2}\left(P_{i}-\frac{P_{\max }}{2}\right)^{2}\right)-\delta_{\max } \sum_{i=j}^{M} \lambda_{j}
$$

Since the cost in the sum of $N$ independent quadratic functions in $P_{i}$ and the constraint set $\mathcal{P}$ is a box, then

$$
\arg \min _{P \in \mathcal{P}} \mathcal{L}_{\theta}(P, \lambda) \Leftrightarrow \arg \min _{0 \leq P_{i} \leq P_{\max }} c_{i} P_{i}+\frac{\theta}{2}\left(P_{i}-\frac{P_{\max }}{2}\right)^{2}
$$

whose solution is unique for each $P_{i}$ and it is given by (11). The minimizer can be rewritten in compact vector form as in (10), where the projector operator is defined as $y=\operatorname{proj}_{\mathcal{P}}(x):=\arg \min _{y \in \mathcal{P}}\|y-x\|$, i.e. the closest point of $\mathcal{P}$ from the vector $x$. Finally note that the optimizer $P^{\theta}(\lambda)$ is well defined for any $\lambda \in \mathbb{R}^{M}$, not only for $\lambda \geq 0$.

Another major advantage of the fact that approximated optimization problem is strongly convex is the following:

Proposition IV.3 For $\theta>0$, the dual function $q_{\theta}(\lambda)$ is continuously differentiable all $\lambda \in \mathbb{R}^{M}$ and its gradient is given by:

$$
\nabla q_{\theta}(\lambda)=B P^{\theta}(\lambda)-\delta_{\max } \mathbf{1}
$$

Moreover, let $\lambda, \lambda^{\prime} \in \mathbb{R}^{M}$, then

$$
\left\|\nabla q_{\theta}(\lambda)-\nabla q_{\theta}\left(\lambda^{\prime}\right)\right\|_{\infty} \leq \frac{\|B\|_{\infty}\|B\|_{1}}{\theta}\left\|\lambda-\lambda^{\prime}\right\|_{\infty}
$$

Proof: From duality theory, it is a well known fact that if $P^{\theta}(\lambda)$ is any minimizer of the Lagrangian, i.e. $P^{\theta}(\lambda) \in$ $\arg \min _{P \in \mathcal{P}} \mathcal{L}_{\theta}(P, \lambda)$, then the vector $g_{\theta}(\lambda):=B P^{\theta}(\lambda)-$ $\delta_{\max } 1$ would be a sub-gradient for the concave function $q_{\theta}(\lambda)=\min _{P \in \mathcal{P}} \mathcal{L}_{\theta}(P, \lambda)=\mathcal{L}_{\theta}\left(P^{\theta}(\lambda), \lambda\right)$, i.e. it has the property that $q_{\theta}\left(\lambda^{*}\right) \leq q_{\theta}(\lambda)+\left(\lambda^{*}-\lambda\right)^{T} g_{\theta}(\lambda)$, i.e. $g_{\theta}(\lambda)$ provides a suitable ascent direction for the concave function $q_{\theta}(\lambda)$. However, since the minimizer $P^{\theta}(\lambda)$ is unique, so it is the sub-gradient $g_{\theta}(\lambda)$. This is a sufficient condition to guarantee that $q_{\theta}(\lambda)$ is strongly convex, continuously differentiable and that $g_{\theta}(\lambda)$ is indeed the exact gradient of $q_{\theta}(\lambda)$, for any $\lambda \in \mathbb{R}^{M}$ (Prop 6.1.1 in [28]). As for the bound on gradient, let us define $\phi(\lambda)=\frac{P_{\max }}{2} \mathbf{1}+\frac{1}{\theta}\left(a-B^{T} \lambda\right)$, then we have:

$$
\begin{aligned}
& \left\|\nabla q_{\theta}(\lambda)-\nabla q_{\theta}\left(\lambda^{\prime}\right)\right\|_{\infty} \leq\left\|B P^{\theta}(\lambda)-B P^{\theta}\left(\lambda^{\prime}\right)\right\|_{\infty} \\
& \leq\|B\|_{\infty}\left\|P^{\theta}(\lambda)-P^{\theta}\left(\lambda^{\prime}\right)\right\|_{\infty} \\
& =\|B\|_{\infty}\left\|\operatorname{proj}_{\mathcal{P}} \phi(\lambda)-\operatorname{proj}_{\mathcal{P}} \phi\left(\lambda^{\prime}\right)\right\|_{\infty} \\
& \stackrel{(1)}{\leq}\|B\|_{\infty}\left\|\phi(\lambda)-\phi\left(\lambda^{\prime}\right)\right\|_{\infty} \\
& =\|B\|_{\infty}\left\|\frac{1}{\theta} B^{T} \lambda-\frac{1}{\theta} B^{T} \lambda^{\prime}\right\|_{\infty} \\
& \leq \frac{1}{\theta}\|B\|_{\infty}\left\|B^{T}\right\|_{\infty}\left\|\lambda-\lambda^{\prime}\right\|_{\infty} \\
& =\frac{1}{\theta}\|B\|_{\infty}\|B\|_{1}\left\|\lambda-\lambda^{\prime}\right\|_{\infty}
\end{aligned}
$$


where inequality (1) follows from the fact that $h: \mathbb{R} \rightarrow \mathbb{R}$ defined in Proposition IV.2 has the property that $\mid h\left(c_{i}\right)-$ $h\left(c_{i}^{\prime}\right)|\leq| c_{i}-c_{i}^{\prime} \mid$, while the other inequalities follows from standard properties of matrix norms.

The previous proposition will be instrumental in guaranteeing the convergence of the iterative algorithm. In particular, the inequality involving the gradient has been obtained for the $\infty$-norm and 1-norm of $B$.

Remark IV.3 An analogous bound holds for the standard 2norm, i.e. $\left\|\nabla q_{\theta}(\lambda)-\nabla q_{\theta}\left(\lambda^{\prime}\right)\right\|_{2} \leq \frac{\|B\|_{2}^{2}}{\theta}\left\|\lambda-\lambda^{\prime}\right\|_{2}$, however the proposed bound is more useful in our scenario. In fact, since $\|B\|_{\infty}=\max _{i} \sum_{j=1}^{N}\left|B_{i j}\right|=\max _{i} \sum_{j \in \mathcal{T}_{i}} B_{i j}$ and $\|B\|_{1}=$ $\max _{j} \sum_{i=1}^{N}\left|B_{i j}\right|=\max _{j} \sum_{i \in \mathcal{S}^{\mathcal{T}}} B_{i j}$, in a typical WPTN the number of neighbours is limited independently of the size of the network. Therefore $\|B\|_{\infty}$ and $\|B\|_{1}$ are likely to be almost independent of the size of the network and upper bounds for their value can be computed off-line.

\section{EMR-SAFe Distributed Power Control ALGORITHM}

The algorithm we propose to solve the approximated optimization problem is given by the following two iterative updates that are performed sequentially:

$$
\begin{aligned}
P^{k+1} & =\operatorname{proj}_{\mathcal{P}}\left(\frac{P_{\max }}{2} \mathbf{1}+\frac{1}{\theta}\left(a-B^{T} \lambda^{k}\right)\right) \\
\lambda^{k+1} & =\lambda^{k}+\rho\left(B P^{k+1}-\delta_{\text {max }} \mathbf{1}\right)
\end{aligned}
$$

Note that as compared to the algorithm proposed in the previous section, the computation of the power level $P^{k+1}$ can be done in closed form by performing only multiplications, sums and thresholding.

Next we discuss the distributed implementation of (12). Let us start by observing that the vector $\lambda$ is composed by the $M$ Lagrange multipliers $\lambda_{1}, \ldots, \lambda_{M}$ where the multiplier $\lambda_{\ell}$ is associated to the constraint $\delta_{\ell} \leq \delta_{\max }$; we assume that $\lambda_{\ell}$ is stored in memory and updated by sensor $S_{\ell}$. Now consider the second equation of (12) and observe that its $\ell$-component can be written as

$\lambda_{\ell}^{k+1}=\lambda_{\ell}^{k}+\rho\left(\sum_{j \in \mathcal{T}_{i}^{\mathcal{S}}} B_{\ell j} P_{j}^{k+1}-\delta_{\max }\right)=\lambda_{\ell}^{k}+\rho\left(\delta_{\ell}^{k+1}-\delta_{\max }\right)$

where $\delta_{\ell}^{k+1}$ is the power measured by sensor $S_{\ell}$ when the transmitters transfer the power $P^{k+1}$. It follows that sensor $S_{\ell}$ can update the value of $\lambda_{\ell}$ in a purely decentralized way just taking periodically the measurements $\delta_{\ell}^{k}, k=1,2, \ldots$, , and without communicating with the other devices.

Now consider the first equation of (12). We have that $P_{i}^{(k+1)}$ can be computed by transmitter $T_{i}$ as in (11) once the value of the quantity $c_{i}$ is known; observe that to compute $c_{i}$, transmitter $T_{i}$ needs to receive the values of the Lagrange multipliers only from the sensors in $\mathcal{S}_{i}^{\mathcal{T}}$, namely, only from the sensors which are within a distance $r$ from transmitter $T_{i}$.

Based on the above observations, we have that the algorithm we propose keeps alternating the following actions (Algorithm 1 presents the pseudocode for the sensor nodes in the system, while Algorithm 2 presents the pseudocode for the transmitter side):
1: After the update of the powers of the transmitters, each sensor $S_{\ell}$ measures the EMR level (Alg. 1, Line 2);

2: Each sensor $S_{\ell}$, based on the taken measurement, updates the Lagrange multiplier $\lambda_{\ell}$ (Alg. 1, Line 3);

3: Each sensor $S_{\ell}$ broadcasts the value of the updated Lagrange multiplier to the transmitters which include $S_{\ell}$ into their transmission coverage (Alg. 1, Line 4);

4: Each transmitter $T_{i}$, based on the received multipliers $\lambda_{\ell}$ from each sensor $S_{\ell} \in \mathcal{S}_{i}^{\mathcal{T}}$ (Alg. 2, Line 8), updates the value of power $P_{i}$ to be transferred completing the step (11) (Alg. 2, Lines 9-10).

5: Each transmitter compensates for the changes in the network topology by estimating the locations of the sensors and receivers periodically and updating the matrices $A$ and

\begin{tabular}{|c|c|c|}
\hline \multicolumn{3}{|c|}{ Algorithm 1 Sensor side $S_{i}$} \\
\hline \multicolumn{3}{|c|}{ Definitions } \\
\hline & $\rho$ & $\triangleright$ a constant \\
\hline & $\triangleright$ a con & resenting EMR threshold \\
\hline & $\triangleright \mathrm{EMR}$ me & nt of sensor $S_{\ell}$ at time $k$ \\
\hline & $\triangleright$ Lagrangian & er of sensor $S_{\ell}$ at time $k$ \\
\hline & $\square$ At each iteration & \\
\hline 2: & Sample $\delta_{\ell}^{k+1}$ & $\triangleright$ Measure EMR value \\
\hline 3: & $\lambda_{\ell}^{k+1}=\lambda_{\ell}^{k+1}+\rho\left(\delta_{\ell}^{k+1}-\delta_{\max }\right)$ & $\triangleright$ Update $\lambda_{\ell}^{k}$ \\
\hline & broadcast $\lambda_{\ell}^{k+1}$ & $\triangleright$ Send to $i \in \mathcal{T}_{\ell}^{\mathcal{S}}$ \\
\hline
\end{tabular}
$B$ (Alg. 2, Lines 1-5).

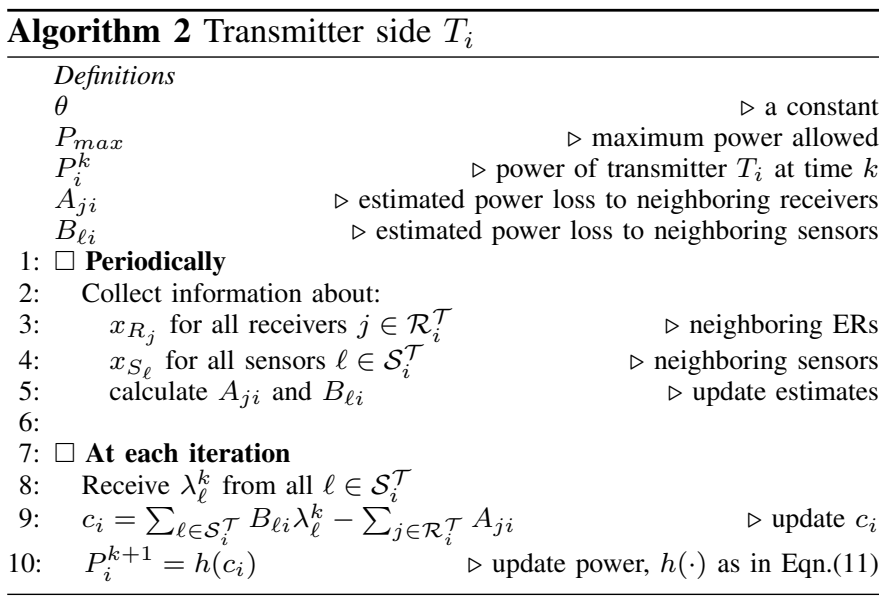

Remark V.1 Observe that in Algorithm described in (12) we do not force the Lagrange multipliers to be non-negative, since the algorithm will guarantee it asymptotically. This follows from the properties of the dual function $q(\lambda)$ stated in Proposition IV.3.

Remark V.2 In order to estimate the locations of the energy receivers and sensors to update the matrices $A$ and $B$ periodically, each transceiver can employ well-known distance estimation and localization techniques, e.g. by using the received signal strength indication, time of arrival/time difference of arrival, angle of arrival, and communication range [29].

\section{A. Convergence analysis for static nodes}

In next proposition we state the convergent properties of Algorithm in (12) under the assumptions that the nodes are static, i.e. the matrices $A$ and $B$ do not change over time. 
Proposition V.1 Consider the algorithm (12). If

$$
\rho \leq \frac{2 \theta}{\|B\|_{\infty}\|B\|_{1}},
$$

then

$$
\lim _{k \rightarrow \infty} P^{k}=P_{\theta}^{*} .
$$

Proof: According to Proposition IV.3, the evolution of the Lagrange multipliers in algorithm (12) can be written as

$$
\lambda^{k+1}=\lambda_{k}+\rho \nabla q_{\theta}\left(\lambda^{k}\right)
$$

Since $q_{\theta}(\lambda)$ is concave, it corresponds to a standard gradientascent algorithm with constant step-size $\rho$. Also, according to Proposition IV.3, its gradient is Lipschitz continuous with constant $L=\frac{\|B\|_{\infty}\|B\|_{1}}{\theta}$. Then, based on Proposition 2.1 in [30], we have that $\lim _{k \rightarrow \infty} q_{\theta}\left(\lambda^{k}\right)=q_{\theta}\left(\lambda^{*}\right)$ and since $q_{\theta}(\lambda)$ is continuous and $\lambda^{*}$ is singleton, then also $\lim _{k \rightarrow \infty} \lambda^{k}=$ $\lambda^{*}$. In turn, also $P^{\theta}(\lambda)$ is continuous in $\lambda$ and therefore $\lim _{k \rightarrow \infty} P^{k}=P^{\theta}\left(\lambda^{*}\right)$. Finally, since there is no duality gap, we necessarily have that $P^{\theta}\left(\lambda^{*}\right)=P_{\theta}^{*}$.

Remark V.3 It is possible to refine the above result providing also some insights into the rate of convergence of the proposed algorithm. In particular it is possible to show that, under the more restrictive assumption $\rho \leq \frac{\theta}{\|B\|_{\infty}\|B\|_{1}}$, it holds (see [28])

$$
\left|q_{\theta}\left(\lambda^{k}\right)-q_{\theta}\left(\lambda^{*}\right)\right| \leq \frac{1}{\rho k}\left\|\lambda^{0}-\lambda^{*}\right\| .
$$

It follows that the rate of convergence is $O(1 / k)$, i.e. is sublinear in log-scale (i.e. sub-exponential in standard scale). One might wonder if such rate can be improved to be linear (i.e. exponential). This typically would require to have $q_{\theta}(\lambda)$ to be strongly concave, i.e. $q_{\theta}\left(\lambda^{\prime}\right) \leq q_{\theta}(\lambda)+\nabla g_{\theta}(\lambda)^{T}\left(\lambda^{\prime}-\right.$ $\lambda)-\epsilon\left\|\lambda^{\prime}-\lambda\right\|^{2}$ for some $\epsilon>0$. However, this is not the case in our scenario due to the presence of the box constraints $P \in \mathcal{P}$ in the primal problem, which prevents that.

Remark V.4 The previous analysis is based on the assumption that the nodes are static and therefore the matrices $A$ and $B$ are constant. If the nodes are mobile, then the proposed algorithm will not reach the optimal instantaneous solution of the LP problem, but it will track such optimal solution since the step-size $\rho$ is constant, differently from [19] in which the step-size is required to go to zero over time.

\section{Numerical Simulations}

We now present the numerical simulations of the proposed algorithm in MATLAB for the sample network depicted in Fig. 2. The network is composed of 25 transmitters, 20 receivers and 5 sensor nodes. We also implemented the centralized LP formulation presented in (5) using MATLAB's linprog function to obtain the optimal solution. The arbitrarily selected parameter values during simulations are presented in Table I. We set the initial powers of the transmitters randomly.

We performed several simulations for the proposed algorithm with different $\theta$ values - by using the corresponding maximum $\rho$ values according to the Proposition V.1. Figure 3

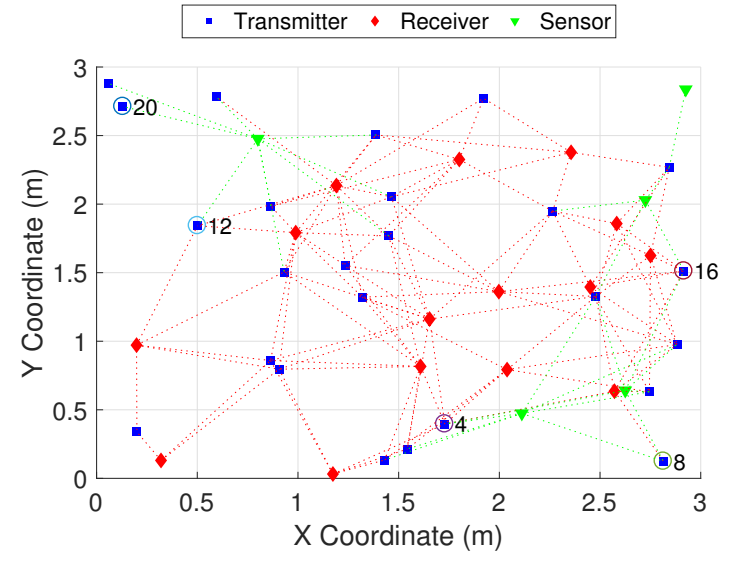

Fig. 2. The random WPT used for the simulations. The transmitters 4, 8, 12, 16 and 20 are marked with circles.

TABLE I

THE PARAMETER VALUES USED DURING OUR EXPERIMENTS.

\begin{tabular}{cccccc}
$\gamma$ & $\mathbf{P}_{\min }$ & $\mathbf{P}_{\max }$ & $\delta_{\max }$ & $\alpha$ & $\beta$ \\
\hline \hline 100 & 0 & 10 & 1 & 1 & 0.1 \\
\hline
\end{tabular}

(top) shows the the maximum EMR overflow in the network, i.e. maximum $\delta_{\ell}$ at each iteration which is normalized with respect to $\delta_{\max }$. As can be observed from this figure, even though the initial network violates the EMR constraints, each iteration of the algorithm forces the network to transition towards a safe state. Observe that the larger values of the $\theta$ led to faster transition time: with $\theta=1$, the EMR overflow is reaching almost $1 \%$ of $\delta_{\max }$ after iteration 200, which is acceptable from practical perspective. Figure 3 (bottom) also depicts the steady-state total transmitted power to the receivers $a^{T} P_{\theta}^{*}$ which is normalized with respect to the optimal LP solution $-J\left(P^{*}\right)-0.90$ is subtracted for the clearness of the plot. The smaller values of the $\theta$ led the cost function of the
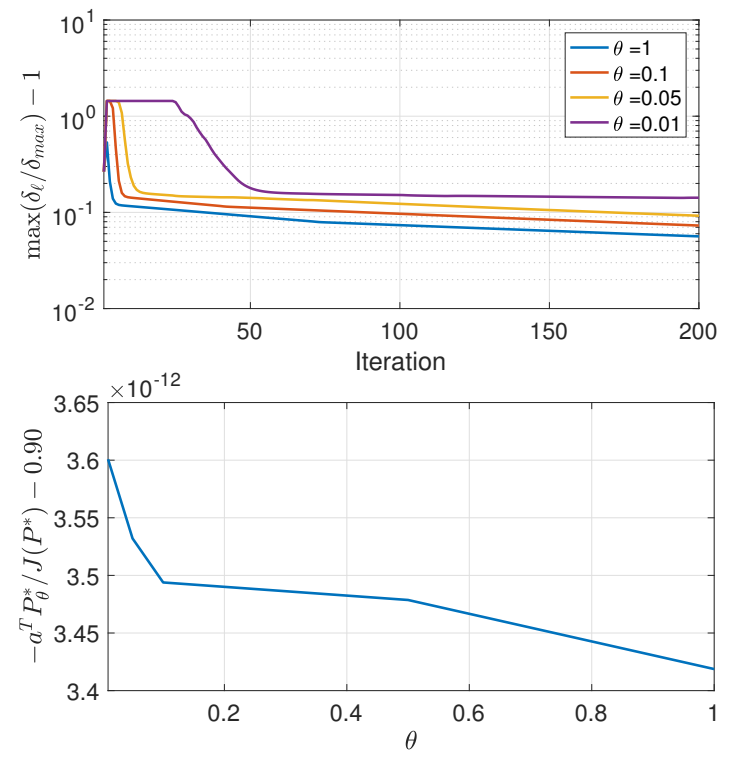

Fig. 3. The EMR overflow (up) and steady-state total transmitted power to the receivers (bottom) using the proposed distributed solution with different $\theta$ and corresponding maximum $\rho$ values according to Proposition V.1. 


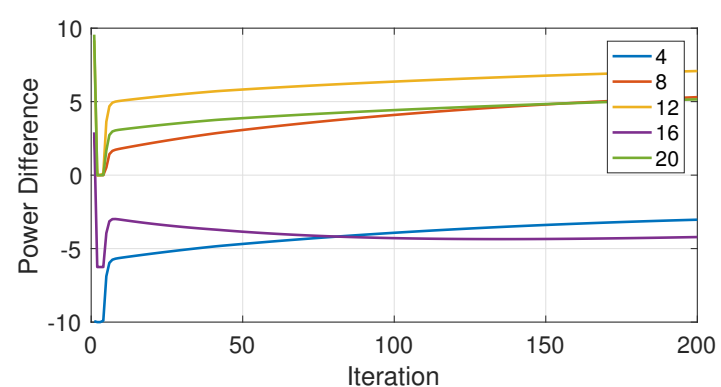

Fig. 4. The difference of the transmitter power levels in our algorithm and their optimal values, with $\theta=0.1$ and the corresponding maximum $\rho$.

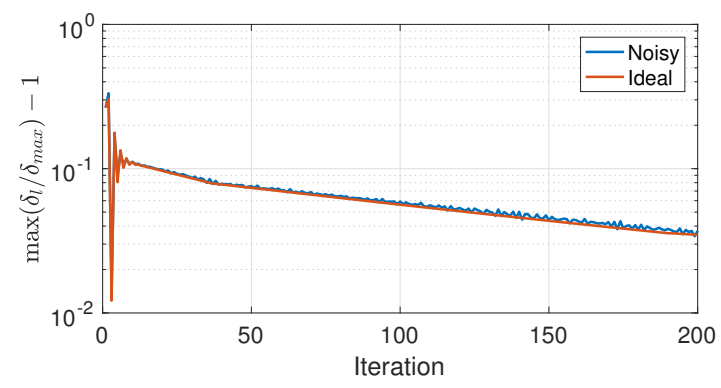

Fig. 5. The difference between the normalized EMR overflows using the ideal and realistic matrices with the proposed algorithm with $\theta=1$.

distributed algorithm closer to the cost function of the optimal LP solution, and in turn more power is transferred. On the other hand, as mentioned previously, the smaller $\theta$ leads to longer transient, which indicates that there is always a tradeoff between faster transient to the safe state and the amount of total power transmitted.

Figure 4 presents the difference of the transmitter power levels $(4,8,12,16$ and 20) and their values with the centralized optimal solution. Observe that the final powers of the transmitters 4, 16 and 20 were greater than their power levels in LP solution, and it was the opposite case for the transmitters 8 and 12. This is due to the fact that there are more than one optimal configurations, and MATLAB finds one among them. It is the case that our algorithm converges to another optimal state asymptotically, in which the power configurations are different. Moreover, our algorithm provides a feasible but suboptimal solution which depends on the selection of $\theta$.

In reality, each transmitter $T_{i}$ in the network implements the following algorithm:

$$
P_{i}^{k}=h\left(c_{i}^{k}\right)=h\left(\sum_{j \in \mathcal{S}_{i}^{\mathcal{T}}} \hat{B}_{j i} \lambda_{j}^{k}-\sum_{j \in \mathcal{R}_{i}^{\mathcal{T}}} \hat{A}_{j i}\right)
$$

since they can only obtain the estimates of the matrices $A$ and $B$, denoted by $\hat{A}$ and $\hat{B}$; respectively. However, the sensors update their Lagrange multipliers by taking EMR overflow measurements; i.e. $\delta_{i}^{k}=B P^{k+1}$ represent the actual EMR value observed by the sensor $S_{i}$. In other words, the update of the Lagrange multipliers is performed using the real matrix $B$. Figure 5 presents the EMR overflows in the ideal and realistic cases where we introduced an uniformly distributed error of $20 \%$ to all of the components of the matrices $A$ and $B$ initially, which resulted in the matrices $\hat{A}$ and $\hat{B}$. At each iteration, we

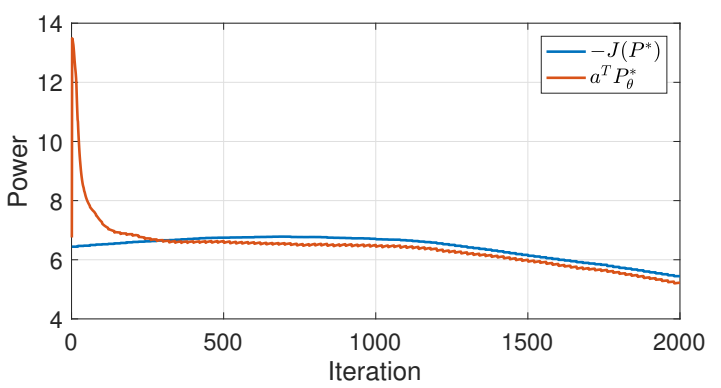

Fig. 6. The effect of mobility in the network. The proposed algorithm tracks the optimal solution.

also introduced a dynamic random error of at most $2 \%$ to these matrices. Observe from the figure that also in the noisy case, the safety constraints are asymptotically satisfied since the Lagrange multipliers are updated by considering actual sensor measurements.

In order to explore the effect of mobility, we considered the deployment area shown in Fig. 2 and created a random network of 50 transmitters, 10 receivers and 150 sensors. The positions of all the nodes are slowly changed according to a random walk: at each iteration we modified the entries of the matrices $A$ and $B$ by adding a random value in the interval $\left[-10^{-4}, 10^{-4}\right]$. Figure 6 depicts the total power transmitted by using the proposed algorithm (with $\theta=0.9$ ) and the centralized linear programming solution. Due to the mobility, the matrices $A$ and $B$ change over time-leading to a different optimal solution at each change in the network topology. It can be observed that after the initial transient necessary to find the optimal solution, the proposed algorithm adapts to these changes and tracks the optimal solution with a small error.

\section{CONClusions ANd Future Work}

In this article, we considered the maximization of the total transmitted wireless power to RF energy harvesting devices subject to the electromagnetic radiation safety constraints. Our main contribution was to introduce a dual ascent-like distributed safe-charging algorithm where energy transmitters communicate only with the sensors in their communication range and adjust their power levels without global information. We provided an extensive theoretical analysis of the proposed approach and presented several numerical simulations in MATLAB that showed that our algorithm satisfies the EMR constraints with a performance comparable to that of the optimal centralized linear programming solution. We would like to leave the implementation of our algorithm on a real hardware platform and its experimentation in a real-world testbed as a future work. It is also interesting to explore the case where each receiver has its individual power requirement and the transmitters are turned-off after the required power is transmitted. In order to eliminate continuous power transmission and in turn energy waste, feedbacks from the receiver devices should be considered. We also would like to explore this case within the context of a future study.

\section{REFERENCES}

[1] Q. Liu, K. S. Yildirim, P. Pawełczak, and M. Warnier, "Safe and secure wireless power transfer networks: challenges and opportunities in rf- 
based systems," IEEE Communications Magazine, vol. 54, no. 9, pp. 74-79, 2016.

[2] S. Gollakota, M. S. Reynolds, J. R. Smith, and D. J. Wetherall, "The emergence of rf-powered computing," Computer, vol. 47, no. 1, pp. 32 39, 2014.

[3] S. Naderiparizi, A. N. Parks, Z. Kapetanovic, B. Ransford, and J. R. Smith, "Wispcam: A battery-free rfid camera," in RFID (RFID), 2015 IEEE International Conference on. IEEE, 2015, pp. 166-173.

[4] Q. Liu, M. Golinński, P. Pawełczak, and M. Warnier, "Green wireless power transfer networks," IEEE Journal on Selected Areas in Communications, vol. 34, no. 5, pp. 1740-1756, 2016.

[5] I. Krikidis, "Simultaneous information and energy transfer in large-scale networks with/without relaying," IEEE Transactions on Communications, vol. 62, no. 3, pp. 900-912, 2014.

[6] X. Lu, P. Wang, D. Niyato, D. I. Kim, and Z. Han, "Wireless charging technologies: Fundamentals, standards, and network applications," IEEE Communications Surveys \& Tutorials, vol. 18, no. 2, pp. 1413-1452, 2016.

[7] A. Costanzo, M. Dionigi, D. Masotti, M. Mongiardo, G. Monti, L. Tarricone, and R. Sorrentino, "Electromagnetic energy harvesting and wireless power transmission: A unified approach," Proceedings of the IEEE, vol. 102, no. 11, pp. 1692-1711, 2014.

[8] V. Talla, B. Kellogg, B. Ransford, S. Naderiparizi, S. Gollakota, and J. R. Smith, "Powering the next billion devices with wi-fi," in Proceedings of the 11th ACM Conference on Emerging Networking Experiments and Technologies. ACM, 2015, p. 4.

[9] S.-N. Daskalakis, A. Georgiadis, A. Bletsas, and C. Kalialakis, "Dual band rf harvesting with low-cost lossy substrate for low-power supply system," in Antennas and Propagation (EuCAP), 2016 10th European Conference on. IEEE, 2016, pp. 1-4.

[10] R. G. Cid-Fuentes, M. Y. Naderi, K. R. Chowdhury, A. CabellosAparicio, and E. Alarcón, "On the scalability of energy in wireless rf powered internet of things," IEEE Communications Letters, vol. 20, no. 12, pp. 2554-2557, 2016.

[11] L. Fu, P. Cheng, Y. Gu, J. Chen, and T. He, "Optimal charging in wireless rechargeable sensor networks," IEEE Transactions on Vehicular Technology, vol. 65, no. 1, pp. 278-291, 2016.

[12] F. Sangare, Y. Xiao, D. Niyato, and Z. Han, "Mobile charging in wireless-powered sensor networks: Optimal scheduling and experimental implementation," IEEE Transactions on Vehicular Technology, 2017.

[13] S. Bi and R. Zhang, "Distributed charging control in broadband wireless power transfer networks," IEEE Journal on Selected Areas in Communications, vol. 34, no. 12, pp. 3380-3393, 2016.

[14] A. Ahlbom, U. Bergqvist, J. Bernhardt, J. Cesarini, M. Grandolfo, M. Hietanen, A. Mckinlay, M. Repacholi, D. Sliney, J. Stolwijk et al., "Guidelines for limiting exposure to time-varying electric, magnetic, and electromagnetic fields (up to $300 \mathrm{GHz}$ )," Health Phys., vol. 74, no. 4, pp. 494-522, Apr. 1998.

[15] C. Kalialakis and A. Georgiadis, "The regulatory framework for wireless power transfer systems," Wireless Power Transfer, vol. 1, no. 2, pp. 108 118, Sep. 2014.

[16] H. Dai, Y. Liu, G. Chen, X. Wu, and T. He, "Safe charging for wireless power transfer," in IEEE INFOCOM 2014-IEEE Conference on Computer Communications. IEEE, 2014, pp. 1105-1113.

[17] S. Nikoletseas, T. P. Raptis, and C. Raptopoulos, "Radiation-constrained algorithms for wireless energy transfer in ad hoc networks," Computer Networks, 2017.

[18] H. Dai, Y. Liu, G. Chen, X. Wu, and T. He, "Scape: Safe charging with adjustable power," in Distributed Computing Systems (ICDCS), 2014 IEEE 34th International Conference on. IEEE, 2014, pp. 439-448.

[19] A. Nedic and A. Ozdaglar, Convex Optimization in Signal Processing and Communications. Cambridge University Press, 2010, ch. Cooperative Distributed Multi-Agent Optimization, pp. 340-386.

[20] S. Boyd, N. Parikh, E. Chu, B. Peleato, and J. Eckstein, "Distributed optimization and statistical learning via the alternating direction method of multipliers," Foundations and Trends $§$ in Machine Learning, vol. 3, no. 1, pp. 1-122, 2011.

[21] R. Carli, G. Notarstefano, L. Schenato, and D. Varagnolo, "Analysis of newton-raphson consensus for multi-agent convex optimization under asynchronous and lossy communications," in Decision and Control (CDC), 2015 IEEE 54th Annual Conference on. IEEE, 2015, pp. 418424.

[22] G. Notarstefano and F. Bullo, "Distributed abstract optimization via constraints consensus: Theory and applications," IEEE Transactions on Automatic Control, vol. 56, no. 10, pp. 2247-2261, 2011.
[23] M. Todescato, G. Cavraro, R. Carli, and L. Schenato, "A robust blockjacobi algorithm for quadratic programming under lossy communications," vol. 48, no. 22. Elsevier, 2015, pp. 126-131.

[24] R. Carli and G. Notarstefano, "Distributed partition-based optimization via dual decomposition," in Decision and Control (CDC), 2013 IEEE 52nd Annual Conference on. IEEE, 2013, pp. 2979-2984.

[25] V. Kekatos and G. B. Giannakis, "Distributed robust power system state estimation," IEEE Transactions on Power Systems, vol. 28, no. 2, pp. 1617-1626, 2013.

[26] K. S. Yildirim, R. Carli, and L. Schenato, "A distributed dual-ascent approach for power control of wireless power transfer networks," in IEEE Conference on Decision and Control (CDC 2017). IEEE, 2017.

[27] K. S. Yıldırım, R. Carli, and L. Schenato, "Distributed control of wireless power transfer subject to safety constraints," IFAC-PapersOnLine, vol. 50, no. 1, pp. 13210-13 215, 2017.

[28] D. P. Bertsekas, Nonlinear programming. Athena scientific Belmont, 1999.

[29] A. Boukerche, H. A. Oliveira, E. F. Nakamura, and A. A. Loureiro, "Localization systems for wireless sensor networks," IEEE wireless Communications, vol. 14, no. 6, 2007.

[30] D. P. Bertsekas and J. N. Tsitsiklis, Parallel and distributed computation: numerical methods. Prentice hall Englewood Cliffs, NJ, 1989, vol. 23.

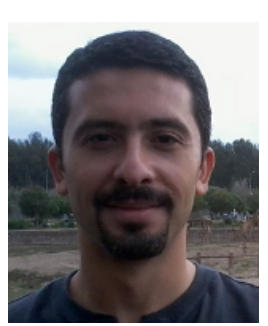

Kasım Sinan Yıldırım received the Ph.D. degree in computer engineering from Ege University, İzmir, Turkey in 2012. He was a visiting scholar at the Department of Information Engineering, University of Padova in 2013. Between 2015-2017, he was a post-doctoral researcher at Embedded Software Group, Delft University of Technology. Currently, he is an assistant professor at the Department of Computer Engineering, Ege University and a research affiliate with the Embedded Software Group at Delft University of Technology. His research interests are wireless embedded systems, embedded software and distributed algorithms.

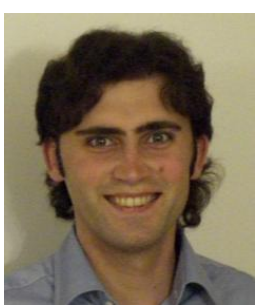

Ruggero Carli received the Laurea Degree in Computer Engineering and the Ph.D. degree in Information Engineering from the University of Padova, Padova, Italy, in 2004 and 2007, respectively. From 2008 through 2010, he was a Post-doctoral Fellow with the Department of Mechanical Engineering, University of California at Santa Barbara. He is currently an Associate Professor with the Department of Information Engineering, University of Padova. His research interests include control theory and, in particular, control under communication constraints, cooperative control, and distributed estimation.

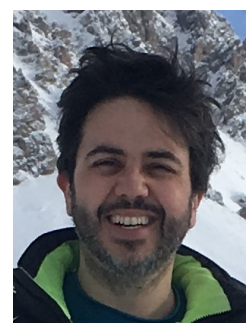

Luca Schenato received the Dr. Eng. degree in electrical engineering from the University of Padova in 1999 and the Ph.D. degree in Electrical Engineering and Computer Sciences from the UC Berkeley, in 2003. He held a post-doctoral position in 2004 and a visiting professor position in 2013-2014 at U.C. Berkeley. Currently he is Associate Professor at the Information Engineering Department at the University of Padova. His interests include networked control systems, multi-agent systems, wireless sensor networks, smart grids and cooperative robotics. Luca Schenato has been awarded the 2004 Researchers Mobility Fellowship by the Italian Ministry of Education, University and Research (MIUR), and the 2006 Eli Jury Award in U.C. Berkeley and the EUCA European Control Award in 2014. He served as Associate Editor for IEEE Trans. on Automatic Control from 2010 to 2014 and he is Fellow of IEEE since 2017. 